Sādhanā Vol. 38, Part 3, June 2013, pp. 377-395. (c) Indian Academy of Sciences

\title{
Effective enhancement of classification of respiratory states using feed forward back propagation neural networks
}

\author{
A BHAVANI SANKAR ${ }^{1, *}$, J ARPUTHA VIJAYA SELVI ${ }^{2}$, \\ D KUMAR $^{3}$ and K SEETHA LAKSHMI ${ }^{1}$
}
${ }^{1}$ Department of Electronics and Communication Engineering, Anjalai Ammal- Mahalingam Engineering College, Kovilvenni 614 403, India
${ }^{2}$ Department of Electronics and Communication Engineering, Kings College of Engineering, Punalkulam, India
${ }^{3}$ Department of Electronics and Communication Engineering, Periyar Maniyammai University, Thanjavur, India
e-mail: absankar72@gmail.com

MS received 28 November 2011; revised 4 February 2013; accepted 22 April 2013

\begin{abstract}
In biomedical signal analysis, Artificial Neural Networks are frequently used for classification, owing to their capability to resolve nonlinearly separable problems and the flexibility to implement them on-chip processor, competently. Artificial Neural Network for a classification task attempts to hand design a network topology and to find a set of network parameters using a back propagation training algorithm. This work presents an intelligent diagnosis system using artificial neural network. Features were extracted from respiratory effort signal based on the threshold-based scheme and the respiratory states were classified into normal, sleep apnea and motion artifacts. The introduced neural classifier was then trained with different back propagation training algorithms and the classified output was compared with the hand designed results. Five different back propagation training algorithms were used for training, such as Levenberg-Marquardt, scaled conjugate gradient, BFGS algorithm, one step secant and Powell-Beale restarts. Our results revealed that the system could correctly classify at an average of $98.7 \%$, when the LM training method was used. Receiver Operating Characteristic (ROC) analysis and confusion matrix showed that the LM method conferred a more balanced and an apt classification of sleep apnea and normal states.
\end{abstract}

Keywords. Sleep apnea; back propagation neural network; Levenberg-Marquardt algorithm; confusion matrix; receiver operating characteristic.

\section{Introduction}

Sleep-disordered breathing (sleep apnea) is associated with an increased threat of demise, in accordance with the new fangled results from the Wisconsin Sleep Cohort, a 18-year

*For correspondence 
observational study supported by the National Heart, Lung, and Blood Institute (NHLBI) of National Institute of Health. Sleep apnea is a prevalent physical disorder, affecting millions of adults and children. It is characterized by a reduction or a pause in breathing during sleep. It involves frequent arousals from sleep - not automatically to complete awakening, because breathing has ceased. It has emerged as a major health problem to date, as so many persons drop sleep due to this condition that their lack of awareness poses a solemn vulnerability. Sleep apnea is related to hypertension and cardiac arrest, and may also cause impaired cerebral functioning, late reaction times, and difficulty in maintaining vigilance and concentration. The scenario will be worse, if this disorder is accompanied with high pressures in the lung arteries, leading to cardiac arrest.

The prominent type of sleep apnea is the obstructive sleep apnea. The obstruction occurs via temporary thwart of the upper airway for breathing. Consequently, the amount of oxygen in blood drops, an adequate amount of oxygen cannot flow into lungs through mouth and nose despite the desperate attempts to take breath. It may be manifested by marked daytime sleepiness and if unnoticed, it leads to heart failure, blood pressure (low and high), brain tumour, blood cancer and lung cancer. Besides, it poses a threat of increasing the hazards of diabetes, and also work-related and driving accidents.

Conventional methods for detection of sleeping disorder rely on the recordings of Electrocardiogram, Electroencephalogram, Electromyogram and respiratory signals. However, the hunch back of these techniques is the requirement of a large number of electrodes for signal recording. Moreover, the electrodes have to be pasted on the skin continuously for effective monitoring. Nevertheless, EEG signals (brain signals) employed for sleep apnea detection are random in nature and exact modelling of EMG signals is very complex. It is evident from the previous studies (Walter Karlen et al 2009) the respiratory signals alone are enough and perform even better than the ECG, EEG and EMG signals, and the recording process eliminates the need for more electrodes to be placed on the skin. Hence, the respiratory signals have been exploited and classified in our work for sleep apnea detection.

\section{Previous work}

At first, we focused on automatic signal classification by extracting the features of the signal from a 1 minute data segment through autoregressive modelling (AR) and other techniques. These features were then compared with the threshold values and introduced into a series of conditions to determine the respiratory signal category for each specific epoch. Many researchers have recommended several methods including alternative approaches, such as engineering diagnostic methods, for detecting the respiratory conditions of patients. Results of a previous research (Walter Karlen et al 2009) indicate that the respiratory signals alone are adequate and achieve even better than the pooled respiratory and Cardio signals. Only a minimum number of electrodes are required for extracting respiratory signals, which enables the people to wear the masks or sensors for several days. Apnea detection based on a spectral method has been proposed exhaustively in Correa et al (2009), whereas in Mendez et al (2007), the possibility of recognizing obstructive sleep apnea based on a beat-by-beat feature of ECG signal is considered. The autoregressive methods and KNN linear classifier have also been discussed in literature. The classification of respiratory signals based on the fuzzy logic system has been proposed in Restrepo et al (2006). The work shows that the zero crossing gives a good estimation of the frequency of apnea with less computational complexity (Vladimir Friedman 1994). The second order autoregressive (AR) model, used to extract the dominant frequency and quantify its 
strength has been explicitly demonstrated by Taikang Ning \& Joseph Bronzino (1989). The ECG signal based respiratory signal classification is investigated by Ruangsuwana et al (2010).

Secondly, we analysed the application of neural network systems against conventional techniques for respiratory signal classification. The artificial neural network can achieve the indispensable conversion and clustering operations routinely and concurrently. Two approaches to classify the ECG biomedical signals are presented by Saad Alshaban \& Rawaa Ali (2010). One is the Artificial Neural Network (ANN) with multilayer perceptron and another is the Fuzzy Logic with Fuzzy Knowledge Base Controller (FKBC). Arjon Turnip et al (2010) have proposed a classification method entailing time-series EEG signals with back propagation neural networks (BPNN). Comparative experiments have been conducted using Bayesian Linear Discriminant Analysis (BLDA) to scrutinize the improvement in classification performance of EEG with the proposed method. An alternative evaluation of Obstructive Sleep Apnea (OSA) based on the ECG signal during sleep time is explained (Rajesh Ghongade \& Ghatol 2007). K-Nearest Neighbour (KNN) supervised learning classifier has been employed as an alternative method for categorizing apnea events from normal ones, on a minute-by-minute basis for each recording. A modification of Levenberg-Marquardt algorithm for MLP neural network learning is depicted in Amir Abolfazl Suratgar et al (2005) and the proposed algorithm has better convergence. Al-Ashmouny \& Ahmed Morsy (2005) presented the results of the clinical evaluation of detection and classification of sleep apnea syndromes. Kannathal \& Rajendra Acharya (2003) have proposed a novel and simple local neural classifier method for the recognition of mental tasks from on-line spontaneous EEG signals. The proposed neural classifier recognizes three mental tasks from on-line spontaneous EEG signals with an accuracy of 70\%. A classification method for respiratory sounds (RSS) in patients with asthma and in healthy subjects is illustrated in Peter Varady et al (2002), wherein the Grow and Learn (GAL) neural network is used for classification. Multilayer perceptrons trained with the back propagation algorithm are tested in the detection and classification tasks and are compared with optimal algorithms resulting from likelihood ratio tests in Michalopoulou et al (1995). The Matlab coding and different back propagation training algorithms for the classification of respiratory signals using neural network has been referred in Sivanandam et al (2006).

The development of a feed forward back propagation neural network model to estimate the resultant center of mass (COM) trajectory in the sagittal plane is illustrated (Batker et al 2003). The COM trajectory is one of the primary outputs of the human postural control system, and is indicative of the system's stability. Neural network performance measurements are discussed in Beirut \& Dobbins (1990), which also includes percent correct, average sum-squared error, receiver operating characteristic (ROC) curve measurements, other measurements based on ROC curve parameters, and the chi-square goodness of fit metric. The study (Anchana Khemphila \& Veera Boonjinig 2011) introduces a classification approach using Multilayer Perceptron (MLP) with Back- Propagation learning algorithm and a feature selection algorithm along with biomedical test values to diagnose heart disease. Farzaneh Keyvanfard (2011), has aimed to increase the classification specificity by using multi classifier system. First, a novel pixel search approach is applied to find a significant region in images. Fuzzy C-means are utilized to determine the clear boundary of tumour. Then, shape and texture features are extracted from the region of interest. The genetic algorithm is applied to select the best feature used for classifiers. Sivakumar (2007) has presented an Artificial Neural Network-based method to classify diabetic retinopathy subjects using visual evoked potential phase spectral periodicity components. The results of Simone et al (2002) conceded by artificial neural network trained and tested using feature extraction techniques, reveal the helpfulness of the clustered discrete wavelet transform method with respect to the renowned techniques of discrete Gabor transform and DWT. The proposed algorithm (Tian 
\& Liu 2005) has been tested with 15 overnight polysomnographic (PSG) records, and with a sensitivity rate of $90.7 \%$ and $80.8 \%$, a specificity rate of $86.4 \%$ and $81.4 \%$ for apnea and hypopnea detection, respectively. The work of Padhy (2011) is based on the extraction of rhythmic components and classification of human brain signals using IEEE standard 1057 algorithm and Support Vector Machine (SVM), respectively. The basic procedure includes acquisition, analysis, and feature extraction of brain signals and the development of a classification system using SVM techniques.

Data system analysis methods for designing collective neural network classifiers are considered (Dorogov Yu \& Lesnykh Yu 2011). It is suggested to use methods of sign graph local balancing and algorithms of system behaviour stereotype selection, for construction of competent areas of local classifiers. In the work (Farideh Ebrahimi et al 2008), an endeavour has been made to classify four sleep stages namely, Awake, Stage 1 + REM, Stage 2 and Slow Wave Stage based on EEG signal. Wavelet transform and ANN have been used for this class. An arrhythmia classification algorithm using dedicated wavelets personalized to individual subjects is proposed (Kim \& Tlacz 2011). The morphological filtering and wavelet transform has been performed in this work. PCA and linear discriminant analysis are utilized to compress the morphological data transformed by the dedicated wavelets. The spatial filling index and time-frequency analysis of heart rate variability signal for syndrome detection was presented (Oliver Faust et al 2004). Renyi's entropy is calculated from the signal in the Wigner-Ville and Continuous Wavelet Transform (CWT) domain. Karpagachelvi et al (2010) discussed several techniques and transformations projected before in literature for feature extraction from an ECG signal. As well, this paper also showed a relative study of different methods proposed by researchers in feature extraction from ECG signal. It is discussed in Sepulveda-Cano et al (2011), the methodology for selecting a set of relevant non- stationary features to increase the specificity of the obstructive sleep apnea detector. In this regard, a time-evolving version of the standard linear multivariate decomposition has been discussed to perform the stochastic dimensionality reduction. Sobh et al (1995) demonstrated the database collection for ECG, Arterial Blood Pressure and Respiration Signal Analysis and also the feature extraction, spectral estimation and parameter quantification. The aim of the study (Víctor Marcos et al 2007) was to assess the ability of neural networks as an assistive tool for the diagnosis of the obstructive sleep apnea syndrome (OSAS). Four spectral features from the estimated power spectral density (PSD) of $\mathrm{SaO}_{2}$ were selected. In addition, three input features were computed from a non-linear analysis of $\mathrm{SaO}_{2}$. Two neural classifiers were assessed: the multilayer perceptron (MLP) network and the radial basis function (RBF) network.

An innovative signal classification method is proposed in the paper (Derong Liu et al 2008) that is competent of distinguishing the subjects with sleep disorders, which cause excessive daytime sleepiness (EDS) from normal control subjects who do not have a sleep disorder based on EEG and pupil size. An artificial neural network (ANN) of modified adaptive resonance theory (ART2) has been used to identify the two groups within a pooled group of subjects including those with OSA and healthy controls. The study (Jafari \& Arabalibeik 2009) aims at designing a system for detecting pulmonary system normal and abnormal functions by using spirometry data and multilayer perception neural networks (MLPNN) and hence, different MLP structures have been tested. It is discussed in Tadahiro Azetsu et al (2008), the feature extraction and signal reconstruction of air and bone conduction voices in the time domain, by applying the independent component analysis (ICA). Two different algorithms such as Multi-threshold Time Analysis and Spectral Analysis for automatically detecting sleep apnea events and performance of the overall system in a sleep study with 20 volunteers is illustrated in Nuria Oliver \& Fernando Flores-Mangas (2007). The paper (Catalina Monica Fira \& Liviu Goras 2008) present 
a new algorithm for electrocardiogram (ECG) signal compression based on local extreme extraction, adaptive hysteretic filtering and Lempel-Ziv-Welch (LZW) coding. The algorithm has been verified using eight of the most frequently normal and pathological types of cardiac beats and a multi-layer perceptron (MLP) neural network trained with original cardiac patterns and tested with reconstructed ones. Shekhar Singh \& Gupta (2011) provide the classification based on feed forward back propagation neural network (FFBPN) for detection of breast cancer object. Twenty six thousands set of cell nuclei were obtained by applying image analysis techniques to microscopic slides. The eight feature dataset was given as an input of the input layer to the FFBPN. It was established that FFBNN gives a faster convergence rate and accurate classification for breast cell nuclei. The paper (Deqiang Li et al 2005) involves the development of a well-organized fuzzy wavelet packet (WP) based feature extraction method for the classification of high-dimensional biomedical signal processing data such as magnetic resonance spectra. Classification results were compared with those obtained from common feature extraction methods in the WP domain.

Results in Aaron Lewicke et al (2008) show that the new HRV signal demonstration obtained in the space created by the feature vector based on Shannon entropy of Mallat component energy sharing gives the finest classifier performance with the ART2 neural structure used in hybrid systems. The method in Kostka \& Tkacz (2004) was tested with simultaneous 8 h ECG and polysomnogram (PSG) determined sleep scores from 196 children enrolled in the collaborative home infant monitoring evaluation (CHIME) study. In Kostka \& Tkacz (2008), Learning vector quantization (LVQ), Multilayer perceptron (MLP), and support vector machines (SVMs) were tested for signal classifications. For the best system structure, different types of wavelets were tested and proposed in Kostka \& Tkacz (2008). The results have shown that the ability of generalization for enriched feature extraction (FE)-SVM based system increased, due to selectively choosing only the most representative features for analysing the AF detection problem. Feature extraction of EEG signals played an important role for classifying spontaneous mental activities in EEG-based brain computer interface (BCI). An improved feature extraction method based on multivariate adaptive autoregressive (MVAAR) models has been proposed and applied to the classification of Motor imagery in Jiang Wang et al (2010). In Estrada et al (2004), three features schemes are proposed for EEG signal: Relative Spectral Band Energy, Harmonic Parameters, and Itakura Distance. Autoregressive modelling was performed for spectral estimation. It then compared the performance of these schemes with the view to select an optimal set of features for specific, sensitive, and accurate Neuro-fuzzy classification of sleep stages. A feed forward neural network was used in six-channel EEG signal classification into one of five classes, which corresponded to the selected tasks in Victor Andrei Muiorescu et al (2003). A simpler topology of the neural network and a reduction of the dimension of layers were achieved due to an autoregressive (AR) model used to represent EEG signals. The network performances were analysed based on the classification rate for the cross-validation set.

\section{Formulation of the proposed work}

The general idea of the proposed work is shown in figure 1. Our work focuses on classification of the respiratory signals using Neural Network. Neural network classifies the respiratory signals into three categories: (i) Normal respiration, (ii) Motion artifacts and (iii) Apnea. The main objective of this work is to analyse the various back propagation training algorithms for classification of the respiratory signals and to diagnose the various ailments. 


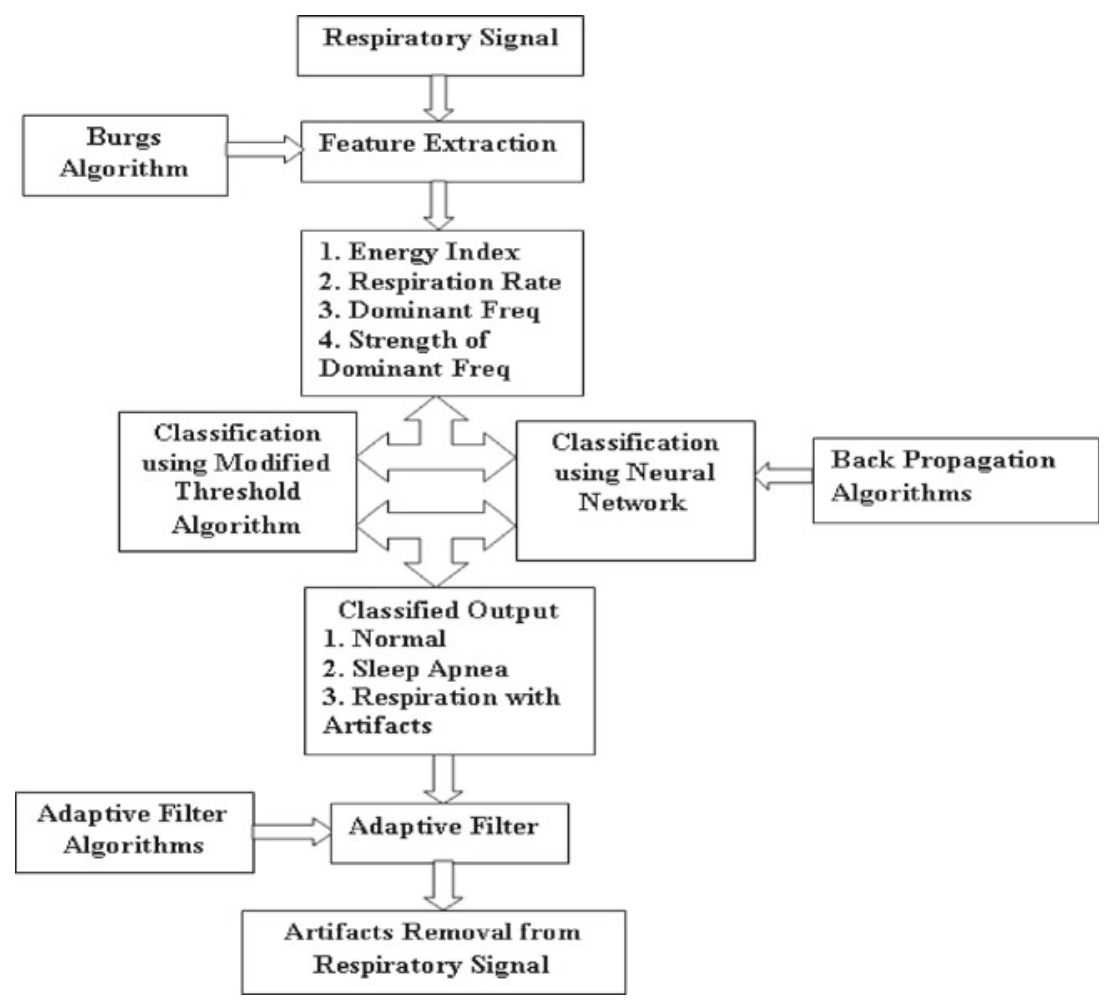

Figure 1. Proposed approach.

\section{Feature extraction}

The four features of the respiratory signals [6] are:

(i) Energy Index (EI)

(ii) Respiration frequency (FZX)

(iii) Dominant frequency estimated by AR modelling (FAR)

(iv) The strength of the dominant frequency estimated by AR modelling (STR).

Classification of respiratory signals was based on the aforementioned four features. These features yielded arithmetic values, which were compared with the threshold values and classifications were made accordingly.

Energy index is,

$$
\mathrm{EI}=\frac{1}{\mathrm{~N}} \sum_{\mathrm{n}=0}^{\mathrm{N}-1}|\mathrm{x}(\mathrm{n})|^{2}
$$

Respiration frequency (FZX) is calculated by counting the number of times that $\mathrm{x}(\mathrm{n})$ crosses a baseline, which is defined as the square root of EI.

$$
\mathrm{FZX}=\sqrt{\mathrm{EI}} .
$$


In order to obtain the features, FAR and STR, the coefficients of a second order AR model have to be estimated. The respiration signal can be modelled as a second order autoregressive model [4] as follows:

$$
X(n)=a_{1} X(n-1)+a_{2} X(n-2)+e(n),
$$

where e (n) is the prediction error and $\left\{a_{1}, a_{2}\right\}$ are AR spectral coefficients. Autoregressive (AR) spectral estimation techniques are known to give better resolution than periodogram methods, when short segments of data are used for analysis. In this work, Burg's algorithm was used for computing AR coefficients. The main advantages of the Burg's method are high frequency resolution and good stability, and less complexity in computation.

Using the second order autoregressive model coefficients, the dominant frequency is calculated as follows,

$$
\mathrm{FAR}=\frac{\mathrm{F}_{\mathrm{s}}}{2 \pi} \arctan \frac{\mathrm{a}_{1}}{\mathrm{a}_{2}}
$$

where Fs is the sampling frequency. A sampling frequency of $250 \mathrm{~Hz}$ was applied in this work.

The strength dominant frequency is determined as follows using the AR spectral coefficients.

$$
\operatorname{STR}=\sqrt{\mathrm{a}_{1}^{2}+\mathrm{a}_{2}^{2}}
$$

The degree of consistency of the respiration rate estimate is determined by STR, which has a value between 0 and 1. For regular rhythm, STR is very close to 1 (as in the case of normal respiration). If STR is too low, then the rate estimates FAR and FZX seem to be unreliable.

In order to make this algorithm healthier, the threshold values of features were obtained as follows. 60 seconds respiratory data (two epochs) were taken and the system performed the same way on these two epochs, and nominal values for each feature were extracted. Then, these nominal values were used to adjust the threshold values as follows [1]: low and high energies were respectively $33 \%$ and $150 \%$ of average energy, low and high frequencies were respectively $50 \%$ and $150 \%$ of nominal frequency and low and high strengths of dominant frequency were respectively $75 \%$ and $95 \%$ of average strength, Which were calculated experimentally.

The classification criteria algorithm is stated in figure 2.

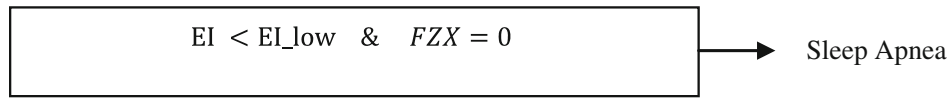

$$
\begin{gathered}
\text { EI }>\text { EI_low \& FZX_min }<F Z X<F Z X \_\max \& \\
\text { STR }<\text { STR_high \& } F A R>F A R \_ \text {min }
\end{gathered}
$$
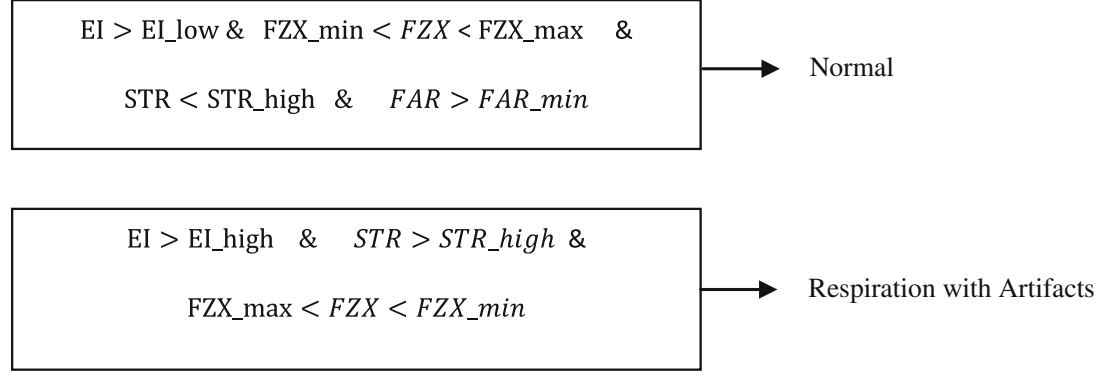

Figure 2. Classification criteria algorithm. 
The human breathing frequency is normally between $0.2-0.3 \mathrm{~Hz}$ and maximum frequency is unlikely to exceed $0.7-0.8 \mathrm{~Hz}$. Hence, these values were adopted as the minimum and maximum threshold values. Square root value of the energy index was used as the proper baseline value for zero crossing, and respiration frequency was deduced from the record of number of times the signal crossed the baseline value. A touching baseline was used to permit changes in the average respiration level. The measured energy index, the respiration rate, the dominant frequency and the strength of the dominant frequency were compared with the preset threshold values and were classified as normal respiration, apnea or respiration with artifacts (Motion artifacts) accordingly.

\section{Neural classifier}

Artificial Neural networks are biologically inspired computer programs to simulate the way in which human brain processes information. It is a very powerful approach for building complex and non-linear relationship between a set of input and output data. Neural Network can be used for moderately correct classification of input data into categories, provided they are previously trained to do so. Usually, neural networks are self-adjusted and trained networks; hence a particular input leads to a specific target output. Such a situation is shown in figure 3. The network is adjusted, based on the comparison between the output and the target, until the network output matches the target. Typically, numerous such input and target pairs are used, in this supervised learning to train a network.

\subsection{Feed forward-back propagation networks}

In a feed forward neural network, neurons are only connected in forward direction. Each layer of the neural network is connected to the next layer, but there is no feedback. Hence, the network is trained using the back propagation training algorithm, a type of supervised training method, and the obtained results are compared with the desired results. The differentiation between the actual results and the desired results is the error. Back propagation is a technique whereby, the weights and input threshold of the neural network are changed in a way that causes this error to be condensed. Back propagation can be used with any feed forward network that uses a differentiable activation function.

A feed forward back propagation neural network consists of two layers. The first layer, or hidden layer, has a tan sigmoid (tan-sig) activation function, and the second layer, or output layer, has a linear activation function. Thus, the first layer limits the output in a narrow range, from which the linear layer can produce all values. The output of each layer can be represented by,

$$
\mathbf{Y}_{N x 1}=\mathrm{f}\left(\mathbf{W}_{N x M} \mathbf{X}_{M, 1}+\mathbf{b}_{N, 1}\right),
$$

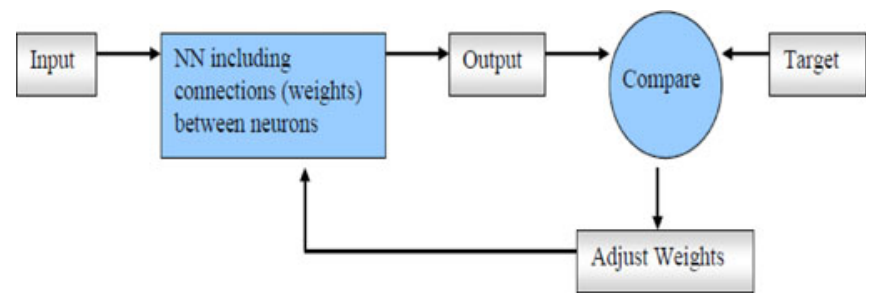

Figure 3. Block diagram for training of neural network. 
where, $\mathbf{Y}$ is a vector containing the output from each of the $\mathrm{N}$ neurons in a given layer, $\mathbf{W}$ is a matrix containing the weights for each of the $\mathrm{M}$ inputs for all $\mathrm{N}$ neurons, $\mathbf{X}$ is a vector containing the inputs, $\mathbf{b}$ is a vector containing the biases and $\mathrm{f}(\cdot)$ is the activation function.

The back propagation algorithm trains a given feed-forward multilayer neural network for a given set of input patterns with known classifications. When each entry of the sample set is presented to the network, the network examines its output response to the sample input pattern. The output response is then compared to the known and desired output and the error value is calculated. Based on the error, the connection weights are adjusted. The back propagation algorithm is based on Widrow-Hoff delta learning rule in which, the weight adjustment is done through mean square error of the output response to the sample input. The set of these sample patterns is repeatedly presented to the network until the error value is minimized.

A feed-forward network with ' $n$ ' input and ' $m$ ' output units, which could consist of any number of hidden units and exhibit any desired feed-forward connection pattern, and a training set $\left\{\left(\mathrm{x}_{1}, \mathrm{t}_{1}\right) \ldots\left(\mathrm{x}_{\mathrm{p}}, \mathrm{t}_{\mathrm{p}}\right)\right\}$ were considered in our work. The training set comprised of ' $\mathrm{p}$ ' ordered pairs of $\mathrm{n}$ - and $\mathrm{m}$-dimensional vectors, which were the input and output patterns. The primitive functions at each node of the network were assumed to be continuous and differentiable. The weights of the edges were real numbers selected at random. When the input pattern xi from the training set was presented to this network, it produced an output $\mathrm{O}_{\mathrm{i}}$ different in general from the target $t_{i}$. We desired to make $o_{i}$ and $t_{i}$ identical for $i=1 \ldots P$, by using learning algorithm. More precisely, we attempted to minimize the error function of the network, defined as,

$$
\mathrm{E}=\frac{1}{2} \sum_{\mathrm{i}=1}^{\mathrm{p}}\left\|\mathrm{o}_{\mathrm{i}}-\mathrm{t}_{\mathrm{i}}^{2}\right\| .
$$

After minimizing this function for the training set, new unknown input patterns were presented to the network and interpolation was expected. The back propagation algorithm was used to find a local minimum of the error function. The network was initialized with randomly chosen weights, and the gradient of the error function was computed and used to correct the initial weights. Our task was to compute this gradient recursively. After choosing the weights of the network to small random values, the back propagation algorithm was used to compute the necessary corrections. The algorithm can be decomposed into the following four steps:

(i) Feed-forward computation

(ii) Back propagation to the output layer

(iii) Back propagation to the hidden layer

(iv) Weight updates.

The algorithm stopped, when the value of the error function had become sufficiently small.

5.1a First step: feed-forward computation: The vector 'o' was presented to the network, and the vectors $\mathrm{o}^{(1)}$ and $\mathrm{o}^{(2)}$ were computed and stored. The evaluated derivatives of the activation functions were also stored in each unit.

5.1b Second step: back propagation to the output layer: The back propagation path from the output of the network to the output unit $\mathrm{j}$ is shown in the B-diagram of figure 4. From this path, all the multiplicative terms which defined the back propagated error $\delta_{j}$ were collected by simple inspection $^{(2)}$. Therefore,

$$
\delta_{\mathrm{j}}^{(2)}=\mathrm{o}_{\mathrm{j}}^{(2)}\left(1-\mathrm{o}_{\mathrm{j}}^{(2)}\right)\left(\mathrm{o}_{\mathrm{j}}^{(2)}-\mathrm{t}_{\mathrm{j}}\right)
$$




\section{backpropagation}

\section{backpropagated error up to unit $j$}

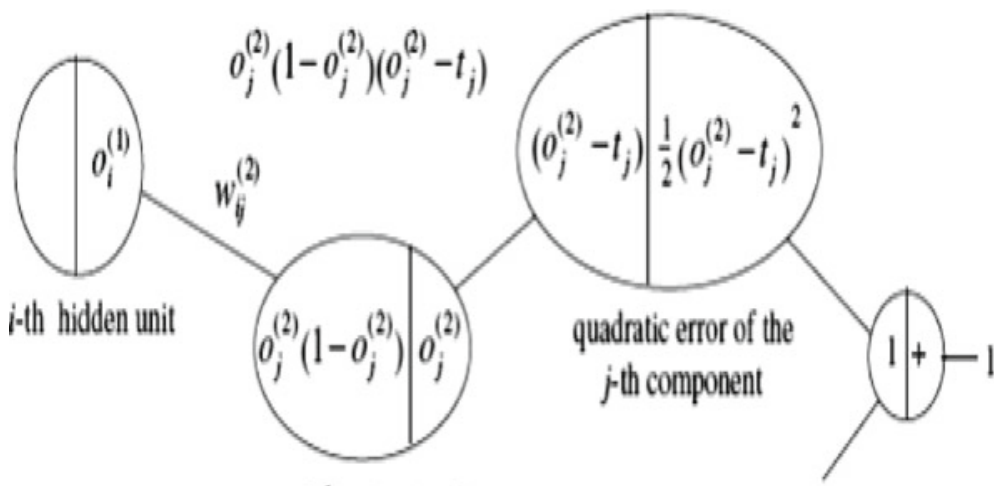

j-th output unit

Figure 4. Back propagation path up to output unit $\mathrm{j}$.

and the partial derivative was,

$$
\frac{\partial \mathrm{E}}{\partial \mathrm{w}_{\mathrm{ij}}^{(2)}}=\left[\mathrm{o}_{\mathrm{j}}^{(2)}\left(1-\mathrm{o}_{\mathrm{j}}^{(2)}\right)\left(\mathrm{o}_{\mathrm{j}}^{(2)}-\mathrm{t}_{\mathrm{j}}\right)\right] \mathrm{o}_{\mathrm{i}}^{(1)}=\delta_{\mathrm{j}}^{(2)} o_{\mathrm{i}}^{(1)}
$$

In the last step, the weight $\mathrm{w}_{\mathrm{ij}}^{(2)}$ was considered to be a variable and its input $\mathrm{o}_{\mathrm{i}}^{(1)}$, a constant. At the input side of the edge with weight $\mathrm{w}_{i j}$, was $\mathrm{o}_{\mathrm{i}}^{(1)}$ and at the output side, the back propagated error $\delta_{j}^{(2)}$.

5.1c Third step: back propagation to the hidden layer: Next, the partial derivatives $\partial \mathrm{E} / \partial \mathrm{w}_{\mathrm{ij}}^{(1)}$ were to be computed. Each unit $\mathrm{j}$ in the hidden layer was connected to each unit $\mathrm{q}$ in the output layer with an edge of weight $\mathrm{wjq}^{(2)}$, for $\mathrm{q}=1 \ldots \mathrm{m}$. The back propagated error $\mathrm{u}$ to unit $\mathrm{j}$ in the hidden layer had to be computed taking into account all possible backward paths. The back propagated error was then,

$$
\delta_{\mathrm{j}}^{(1)}=\mathrm{o}_{\mathrm{i}}^{(1)}\left(1-\mathrm{o}_{\mathrm{j}}^{(1)}\right) \sum_{\mathrm{q}=1}^{\mathrm{m}} \mathrm{w}_{\mathrm{jq}}^{(2)} \delta_{\mathrm{q}}^{(2)} .
$$

Therefore, the partial derivative was,

$$
\frac{\partial \mathrm{E}}{\partial \mathrm{w}_{\mathrm{ij}}^{(1)}}=\delta_{\mathrm{j}}^{(1)} \mathrm{o}_{\mathrm{i}}
$$

The back propagated error had to be computed in the same way for any number of hidden layers and the expression for the partial derivatives of E kept the same analytic form. 
5.1d Fourth step: weight updates: After computing all the partial derivatives, the network weights were updated in the negative gradient direction. Learning constant, $\gamma$ defined the step length of the correction. The corrections for the weights were given by

$$
\Delta \mathrm{w}_{\mathrm{ij}}^{(2)}=-\gamma \mathrm{o}_{\mathrm{i}}^{(2)} \delta_{\mathrm{j}}^{(2)}, \text { for } \mathrm{i}=1, \ldots, \mathrm{k}+1 ; \mathrm{j}=1, \ldots \mathrm{m}
$$

and

$$
\Delta \mathrm{w}_{\mathrm{ij}}^{(1)}=-\gamma \mathrm{o}_{\mathrm{i}} \delta_{\mathrm{j}}^{(1)}, \text { for } \mathrm{i}=1, \ldots, \mathrm{n}+1 ; \mathrm{j}=1, \ldots \mathrm{k},
$$

where we used the convention that $\mathrm{o}_{\mathrm{n}+1}=\mathrm{o}_{\mathrm{k}+1}^{(1)}=1$.

It had been very important to make the corrections to the weights only after the back propagated error had been computed for all the units in the network. Otherwise, the corrections became intertwined with the back propagation of the error and the computed corrections correspond no more to the negative gradient direction.

We initialized the network with random weights and biases, and it was then trained using the different minimization algorithms such as Gradient descent, Variable learning rate, Resilient back propagation, Conjugate gradient, Quasi Newton and Levenberg-Marquardt algorithms. The neural network performance in our classification process was evaluated by means of the following four performance indices:

(i) MSE ('Mean Square Error'), was computed by taking the differences between the target and the actual neural network outputs, squaring them and averaging over all classes and internal validation samples.

(ii) Given a classifier and a set of instances (the test set), a two-by-two 'confusion matrix' was constructed, representing the dispositions of the set of instances.

(iii) A 'ROC curve', which was independent of class distribution or error. ROC curves provided a visual tool for examining the trade-off between the capability of a classifier to identify correctly the positive and negative cases that were incorrectly classified. The more each curve hugged the left and the top edges of the plot, the better was the classification.

(iv) By the use of 'regression line' or equation, scores of the dependent variable were predicted from those of the independent variable. The $\mathrm{R}$ value was a sign of the relationship between the outputs and targets.

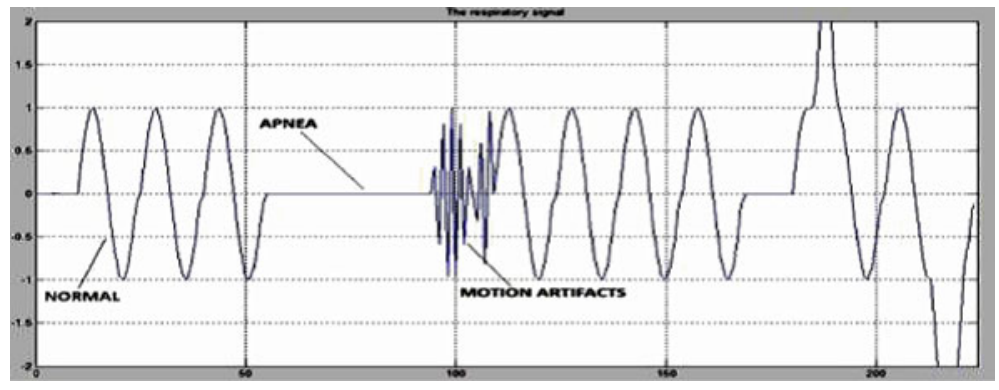

Figure 5. Human respiratory signal. 


\section{Simulation results and discussion}

\subsection{Classification using automatic classification algorithm}

The input to our classification algorithm was a human respiratory signal of $1 \mathrm{~Hz}$, as shown in figure 5. The data segment of the respiratory signal was collected from the MIT-BIH database in the form of data points. These data points were given to the classification algorithm as input.

The signal was sampled at $250 \mathrm{~Hz}$ and a total of 1200 data points were subjected to analysis. The threshold values of the features to be extracted from the signal were provided as the optimum values in the program used for signal classification. The nominal values and threshold values are given in tables 1 and 2 using two epochs.

The respiration data were divided into 20 second epochs and manually scored for comparison. The epochs were then processed by the automatic classification algorithm and compared to manual classification. The classified results provided in table 3 show that the proposed algorithm was capable of classifying with the accuracy of $100 \%$, with respect to normal and sleep apnoic signals. Few discrepancies were encountered with the detection of motion artifacts and hence, 15 sections were identified as unclassified signals.

Table 1. Calculated nominal values to decide threshold.

\begin{tabular}{lcccccc}
\hline Sample & E_Low & E_High & STR_Low & STR_High & FAR_Min & FAR_Max \\
\hline Normal_1 & 0.48 & 2.01 & 0.25 & 0.22 & 0.52 & 1.62 \\
Normal_2 & 0.55 & 2.5 & 0.32 & 0.35 & 0.65 & 1.91 \\
Apnea_1 & 0.3 & 1.25 & 0.15 & 0.2 & 0.41 & 1.35 \\
Apnea_2 & 0.25 & 0.9 & 0.08 & 0.1 & 0.39 & 1.2 \\
Artifacts_1 & 0.70 & 3.12 & 0.5 & 0.52 & 0.75 & 2.22 \\
Artifacts_2 & 0.95 & 4.5 & 0.35 & 0.45 & 0.92 & 2.62 \\
\hline
\end{tabular}

Table 2. Threshold values.

\begin{tabular}{lccc}
\hline Features & Normal & Apnea & Artifacts \\
\hline E_Low & 0.45 & 0.25 & 0.75 \\
E_High & 2.5 & 1.2 & 3.85 \\
STR_Low & 0.22 & 0.05 & 0.38 \\
STR_High & 0.35 & 0.12 & 0.52 \\
FAR_Min & 0.55 & 0.35 & 0.75 \\
FAR_Max & 1.88 & 1.35 & 2.5 \\
\hline
\end{tabular}

Table 3. Classifier output based on threshold.

\begin{tabular}{lcc}
\hline Episode & Manual & Simulation \\
\hline Normal & 730 & 730 \\
Sleep Apnea & 152 & 152 \\
Artifact & 303 & 303 \\
Unclassified & 15 & 15 \\
\hline
\end{tabular}


Table 4. Specifications of neural network.

\begin{tabular}{lll}
\hline Sl.No & \multicolumn{1}{c}{ Parameters } & \multicolumn{1}{c}{ Value } \\
\hline 1 & Type of network & Feed forward back propagation \\
2 & No. of neurons in the input layer & 4 \\
3 & No. of neurons in the hidden layer & 20 \\
4 & No. of neurons in the output layer & 3 \\
5 & Performance function & MSE \\
6 & Activation function in the hidden layer & tan-sigmoid \\
7 & Activation function in the output layer & purelin \\
8 & Learning rate & 0.001 \\
9 & Maximum no. of epochs & 1000 \\
10 & Minimum MSE value & 0 \\
11 & Training parameters ratio & $60 \%$ \\
12 & Validation and testing parameters ratio & $20 \%$ each \\
\hline
\end{tabular}

\subsection{Classification results using neural network}

A synthetic respiratory signal of $1 \mathrm{~Hz}$ was utilized for simulation. The signal was sampled at $20 \mathrm{~Hz}$, and the four main features of the respiratory signal were extracted using a modified threshold based algorithm, which was devised as a part of our research work. A total of 300 synthetically generated samples with four feature sets were used for simulation. The neural network and the training algorithm were implemented with the Matlab. The network had been created using the command 'newff' and the training algorithms were represented as functions in Matlab such as trainlm, trainscg, trains, trainbfg and traincgf. Specifications of the neural network employed for the classification of respiratory signals are given in table 4 .

6.2a Selection of parameters for sleep apnea detection: Initial weights and biases: It influences whether the net reaches a global minima of the error and if so, how rapidly it converges. If the initial weight is too large, the initial input signals to each hidden or output unit will fall in the saturation region, where the derivative of the sigmoid has a very small value. If the initial weights are too small, the net input to a hidden or output unit will approach zero, which then causes extremely slow learning. Hence to get the best results, we chose the initial weights and biases between -1 and +1 .

Number of training pairs: The number of training pairs also plays a key role during training of the nets. To train the networks, the data are divided into three sets: (1) training, (2) validation, and (3) test. The training set (TR) contained the data used to update the synaptic weights. The performance of the network is evaluated on the validation set (VA), and the test set (TE) is used to measure the performance of the network after the training. The training ratio is decided based on repeated iterations with different training-testing ratio. Based on the results in table 5, it was decided to choose $60 \%$ for training, $20 \%$ for testing and $20 \%$ for validation.

Number of hidden neurons: Deciding the number of neurons in the hidden layers is the vital part in deciding the overall neural network architecture. Although these layers do not directly interact with the external environment, they have a tremendous influence on the final output. Both the number of hidden layers and the number of neurons in each of these hidden layers must be carefully considered. Underfitting occurs when there are too few neurons in the hidden layers to adequately detect the signals in a complicated data set. Overfitting occurs when the neural 
Table 5. Classification result for different training pairs.

\begin{tabular}{lcc}
\hline $\begin{array}{l}\text { Training } \\
\text { data } \%\end{array}$ & $\begin{array}{c}\text { Classification } \\
\text { accuracy } \%\end{array}$ & $\begin{array}{c}\text { Elapsed } \\
\text { time (s) }\end{array}$ \\
\hline 10 & 95 & 0.48 \\
20 & 96 & 0.53 \\
30 & 96.7 & 0.57 \\
40 & 97.3 & 0.54 \\
50 & 98.7 & 0.73 \\
60 & 98.7 & 0.59 \\
70 & 97.7 & 0.59 \\
80 & 99.7 & 1.16 \\
90 & 99.3 & 0.76 \\
99 & 100 & 1.85 \\
\hline
\end{tabular}

Table 6. Classification results for different number of hidden neurons.

\begin{tabular}{lccc}
\hline $\begin{array}{l}\text { No. of hidden } \\
\text { neurons }\end{array}$ & $\begin{array}{c}\text { Classification } \\
\text { accuracy } \%\end{array}$ & $\begin{array}{c}\text { Mean square } \\
\text { error }\end{array}$ & $\begin{array}{c}\text { No. of } \\
\text { epochs }\end{array}$ \\
\hline 5 & 97.3 & 0.0086 & 26 \\
10 & 99 & 0.0304 & 23 \\
15 & 98.3 & 0.0421 & 14 \\
20 & 98.7 & 0.0131 & 13 \\
\hline
\end{tabular}

network has so much information processing capacity that the limited amount of information contained in the training set is not enough to train all of the neurons in the hidden layers. Based on the results in table 6, we have decided to choose the number of hidden neurons as 20 .

The classification accuracy was calculated as the ratio of the number of samples correctly classified to the total number of samples. This is verified in the following figures, which contain the plots of the mean square error versus the number of epochs for several algorithms. First, we trained the network to classify the respiratory signals using LM algorithm. The plots of mean square error (MSE) and Receiver operating characteristic curves are shown in figures $6 a$ and b.

The Confusion matrix showing a $98.7 \%$ precision, after 13 training epochs and a mean square error of 0.0453 is shown in figure $7 \mathrm{a}$. The regression plots for training, testing and validation are shown in figure $7 \mathrm{~b}$. The diagonal cells exhibit the number of residue positions that are correctly classified for each structural class. The off-diagonal cells show the number of residue positions that are misclassified. The blue cells portray the total percentage of correctly predicted residues and the total percentages of incorrectly predicted residues are shown in red.

The three axes represent the training, testing and validation data. The dashed line in each axis represents the perfect result - outputs $=$ targets. The hard line represents the best fit linear regression line between outputs and targets. The relation between output and target is indicated by $R$ value. If $R$ value is one, then there is an exact linear relationship between output and target. If $\mathrm{R}$ is close to zero, then there is no linear relationship between output and target. The training data indicated a good fit. The validation and test results also showed $\mathrm{R}$ values greater than 0.9 . 


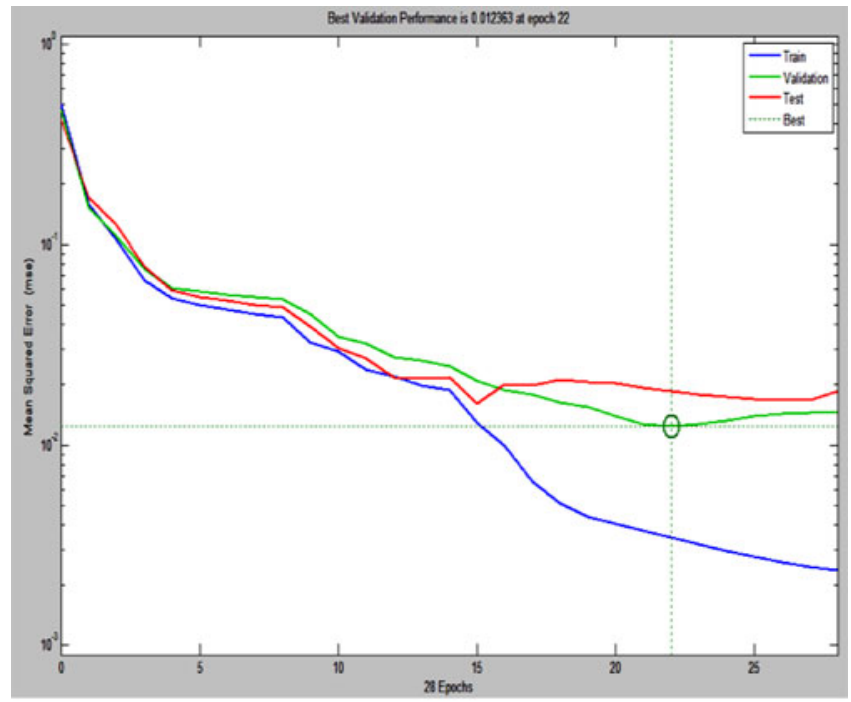

(a)

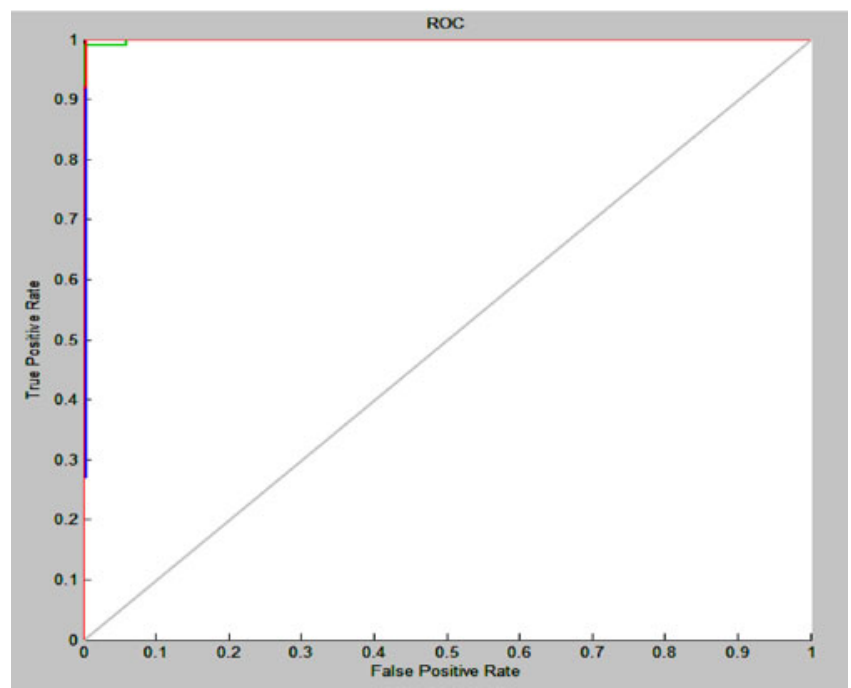

(b)

Figure 6. (a) Plot of MSE. (b) Plot of ROC using LM algorithm.

Then, we repeated the training using scaled conjugate gradient, quasi newton BFGS algorithm, and Powell-Beale restarts and one step secant algorithm. Comparative evaluation done for all the algorithms is tabulated in table 7.

6.2b LM algorithm and Sleep Apnea detection: Levenberg Marquardt (LM) algorithm is a nonlinear optimization algorithm, which uses the Hessian matrix to perform better estimations on both step sizes and directions, so that they can converge much faster than first order algorithms. By combining the training speed of Newton algorithm and the stability of Error Back 


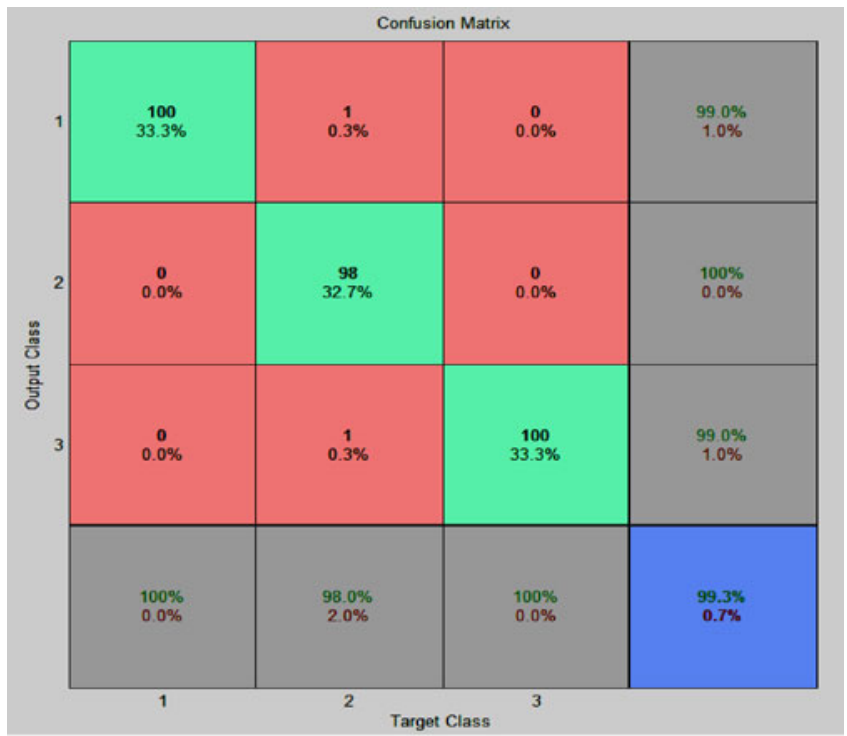

(a)
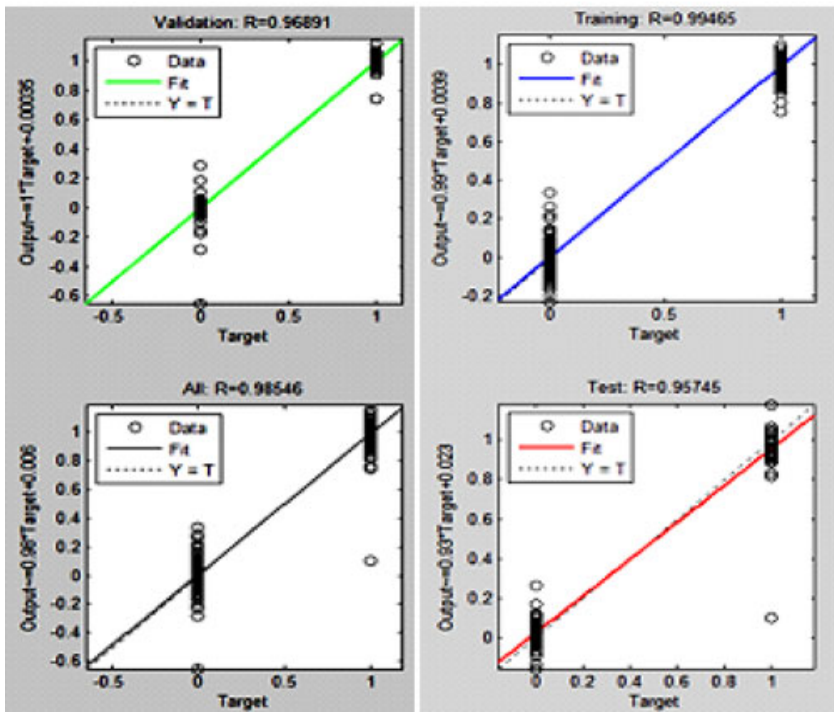

(b)

Figure 7. (a) Confusion matrix, (b) regression plots for training, testing and validation.

Propagation (EBP) algorithm, LM algorithm is regarded as one of the most efficient algorithms for training biomedical signals such as EEG, ECG and respiratory signals. The respiratory signal reflects the electrical activity of respiration and the information on human health and therefore prompts an early diagnosis of any cardiac defect and appropriate treatment.

It is evident that no algorithm has its own order in terms of suitability; there is always a trade-off between the algorithm and application. In this work, a set of apnoic signals is trained with five different back propagation training algorithms such as Levenberg-Marquardt (LM), 
Table 7. Comparative evaluation.

\begin{tabular}{lcccc}
\hline $\begin{array}{l}\text { Training } \\
\text { functions }\end{array}$ & Epochs & Regression & Accuracy & MSE \\
\hline LM & 31 & 0.98286 & 98.7 & 0.013193 \\
OSS & 28 & 0.31804 & 95.7 & 0.069286 \\
BFG & 27 & 0.34170 & 96.3 & 0.059375 \\
SCG & 51 & 0.91973 & 96.7 & 0.041332 \\
CGF & 27 & 0.33979 & 96.3 & 0.057366 \\
\hline
\end{tabular}

Scaled Conjugate Gradient (SCG), BFGS algorithm, One Step Secant and Powell-Beale restarts. Experimental results substantiated that LM algorithm is more suitable for sleep apnea screening than others, because of its high accuracy (98.7) and very low MSE (0.013193). A smaller MSE value indicated that the residuals were small, meaning that the particular MLP had fitted the data well. These results were validated with MATLAB NN tool box, as tabulated in table 7 .

The accuracy of the algorithm will depend on many factors, including the complexity of the problem, the number of data points in the training set, the number of weights and biases in the network, the error goal, and whether the network is being used for pattern recognition or function approximation. The proposed Levenberg-Marquardt approach exhibited a superior performance in terms of classification accuracy and was also easier and simpler to implement and use.

\section{Conclusion and future work}

In our research work, an automatic system for classification of the respiratory states using a modified threshold based algorithm has been developed. The algorithm used four main features extracted from the respiratory signal as its input. The proposed algorithm was capable of classifying normal and sleep apnoic signals with $100 \%$ accuracy. Few disagreements were encountered with the detection of motion artifacts and hence, 15 sections were termed as unclassified signals. To overcome the disagreements and for further enhancement in the classification accuracy, ANN techniques for decision making were developed and implemented. The decision-making was performed using features extracted from respiratory signals. Emphasis was placed on the selection of characteristic features and the accurate extraction of these features. From the results, it could be visualized that the Levenberg-Marquardt back propagation algorithm has provided an excellent performance for the studied application. The performance and trade-offs among the five training methods were also studied. The choice of the algorithm to be used is a trade-off between the performance, convergence rate and training input. The proposed Levenberg-Marquardt approach exhibited a superior performance in terms of classification accuracy and was also easier and simpler to implement and use.

Presently, we are in the progress of designing a processor called soft-core processor, with the algorithm developed in this work and are to be run on an FPGA.

\section{References}

Aaron Lewicke, Sazonov E, Corwin M J, Neuman M and Schuckers S 2008 Sleep versus wake classification from heart rate variability using computational intelligence: consideration of rejection in classification models. IEEE Trans. Biomed. Eng. 55(1): 108-118 
Al-Ashmouny Khaled M and Ahmed Morsy A 2005 Sleep apnea detection and classification using fuzzy logic: clinical evaluation. Proc. IEEE: 6132-6135

Amir Abolfazl Suratgar, Mohammad Bagher Tawakoni and Abbas Hoseinabadi 2005 Modified LevenbergMarquardt method for neural networks training. WASAET 6: 636-638

Anchana Khemphila and Veera Boonjinig 2011 Heart disease classification using neural network and feature selection. Proc. IEEE: 406-409

Arjon Turnip, Keum-Skik, Hong and Shuzhi Sam G E 2010 Back propagation neural networks training for single trial EEG classification. Proc. IEEE: 2462-2467

Batker A L, Szturn T and Moussavi Z 2003 Application of feed forward back propagation neural network to center of mass estimation for use in a clinical environment. Proc. IEEE 3: 2714-2717

Beirut Russell C and Dobbins Roy W 1990 Neural network performance metrics for biomedical applications. Proc. IEEE. Int. Symp: 282-289

Catalina Monica Fira and Liviu Goras 2008 An ECG signals compression method and its validation using NNs. IEEE Trans. Biomed. Eng. 55(4): 1319-1326

Correa Lorena S, Laciar E, Mut V, Torres A and Jane R 2009 Sleep apnea detection based on spectral analysis of three ECG - derived respiratory signals. Proc. IEEE 31: 4723-4726

Deqiang Li, Pedrycz W and Pizzi N J 2005 Fuzzy wavelet packet based feature extraction method and its application to biomedical signal classification. IEEE Trans. Biomed. Eng. 52(6): 1132-1139

Derong Liu, Pang Z and Lloyed S R 2008 A neural network method for detection of obstructive sleep apnea and narcolepsy based on pupil size and EEG. IEEE Transactions on Neural Networks 19(2): 308-318

Dorogov Yu A and Lesnykh Yu V 2011 Neural network classification in non homogeneous feature space. Optical Memory and Neural Networks (Information Optics) 20(2): 120-131

Estrada E, Pang Z and Lloyed S R 2004 EEG feature extraction for classification of sleep stages. Proc. IEEE: 196-199

Farideh Ebrahimi, Mohammad Mikaeili, Edson Estrada and Homer Nazeran 2008 Automatic sleep stage classification based on EEG signals by using neural networks and wavelet packet coefficients. Proc. IEEE: $1151-1154$

Farzaneh Keyvanfard 2011 Feature selection and classification of breast MRI lesions based on Multi classifier. Proc. IEEE Int. Symp: 54-58

Jafari S and Arabalibeik H 2009 Classification of normal and abnormal respiration patterns using flow volume curve and neural network. Proc. IEEE: 110-113

Jiang Wang, Xu Guizhi Z, Wang Lei and Zhang 2010 Feature extraction of brain-computer interface based on improved multivariate adaptive autoregressive models. Proc. BMEI: 895-898

Kannathal N and Rajendra Acharya U 2003 Classification of cardiac patient states using artificial neural networks. J. Clinical Cardiology 8(4): 206-211

Karpagachelvi S, Arthanari M and Sivakumar M 2010 ECG feature extraction techniques - a survey approach. IJCSIS 8(1): 76-80

Kim P and Tlacz E J 2011 An arrhythmia classification algorithm using a dedicated wavelet adapted to different subjects. Biomed. Eng. Online 10(56): 2-19

Kostka P and Tkacz E J 2004 An improvement of unsupervised hybrid biomedical signal classifiers by optimal feature extraction in system preliminary layer. Proc. IEEE: 279-282

Kostka Pawel S and Tkacz Ewaryst J 2008 Feature extraction for improving the support vector machine biomedical data classifier performance. Proc. Int. Symp: 362-363

Mendez Martin O, David D Ruini, Omar P Villantieri and Matteo Matteucci 2007 Detection of sleep apnea from surface ECG based on features extracted by an autoregressive model. Proc. IEEE 29: 6105-6108

Michalopoulou Z H, Nolte L W and Alexandrou D 1995 Performance evaluation of multilayer perceptrons in signal detection and classification. IEEE Trans. Neural. Net. 6(2): 381-386

Nuria Oliver and Fernando Flores-Mangas 2007 Health gear: automatic sleep apnea detection and monitoring with a mobile phone. J. Commun. 2(2): 1-9

Oliver Faust, Rajendra Acharya U, Krishnan S M and Lim C Min 2004 Analysis of cardiac signals using spatial filling index and time-frequency domain. BioMedical Engineering OnLine 3(30): 1-11

Padhy P K 2011 Feature extraction and classification of brain signal. WASET 55: 651-652 
Peter Varady, Tamas Micsik, Sandor Bendek and Zoltan Benyo 2002 A novel method for the detection of apnea and hypopnea events in respiration signals. IEEE Trans. Biomedical Eng. 49(9): 936-942

Rajesh Ghongade and Ghatol A A 2007 A brief performance evaluation of ECG feature extraction techniques for artificial neural network based classification. Proc. IEEE: 1-4

Restrepo M I, Bhandari S and Taikang Ning 2006 Classification of respiration episodes using fuzzy logic. Proc. IEEE 32: 133-134

Ruangsuwana R, Velikic G and Bocko M 2010 Methods to extract respiration information from ECG signals. Proc. IEEE: $570-573$

Saad Alshaban and Rawaa Ali 2010 Using neural and fuzzy software for the classification of ECG signals. Research J. Appl. Sci. Eng. Tech. 2(1): 5-10

Sepulveda-Cano L M, Gil E, Laguma P and Castellanos-Dominguez 2011 Selection of non stationary dynamic features for obstructive sleep apnea detection in children. EURASIP J. Adv. Sign. Proc. 53831410: $1-10$

Shekhar Singh and Gupta P R 2011 Breast cancer detection and classification using neural network. Int. J. Adv. Eng. Sci. Tech. 6(1): 4-9

Simone G, Morabito F C, Polikar R, Ramuhalli P, Udapa L and Udapa S 2002 Feature extraction techniques for ultrasonic signal classification. Int. J. Appl. Electrom. Mech. 15(2001/2002): 291-294

Sivakumar R 2007 Neural network based diabetic retinopathy classification using phase spectral periodicity components. ICGST-BIME Journal 7(1): 23-28

Sivanandam S N, Sumathi S and Deepa S N 2006 Introduction to neural networks using Matlab 6.0., Chapter 15, Tata McGraw-Hill

Sobh J F, Risk M, Barbieri R and Saul J P 1995 Database for ECG, arterial blood pressure, and respiration signal analysis: feature extraction, spectral estimation, and parameter quantification. Proc. IEEE 2: 955-956

Tadahiro Azetsu, Eiji Uchino, Ryosuke Kubota and Noriaki Suetake 2008 Feature extraction and signal reconstruction of air and bone conduction voices by independent component analysis. Proc. IMECS(I): $19-21$

Taikang Ning and Joseph D Bronzino 1989 Automatic classification of respiratory signals. Proc. IEEE: 669-670

Tian J Y and Liu J Q 2005 Apnea detection based on time delay neural network. Proc. IEEE: 609-619

Victor Andrei Muiorescu, Serban M and Lazar A M 2003 Classification of EEG signals represented by AR models for cognitive tasks - a neural network based method. Proc. IEEE Int. Symp. 2: 441-444

Víctor Marcos J, Hornero R, Alvarez D, Del Campo F and Lopez M 2007 Applying neural network classifiers in the diagnosis of the obstructive sleep apnea syndrome from nocturnal pulse oximetric recordings. Proc. IEEE: 5174-5177

Vladimir Friedman 1994 A zero crossing algorithm for the estimation of the frequency of a single sinusoid in white noise. IEEE Trans. Sig. Process. 42(6): 1565-1569

Walter Karlen, Claudio Mattiussi and Dario Floreano 2009 Sleep and wake classification with ECG and respiratory effort signals. IEEE Trans. Biomed. Circuits Syst. 3(2): 71-78 